This work is licensed under a Creative Commons "Attribution 4.0 International" license.

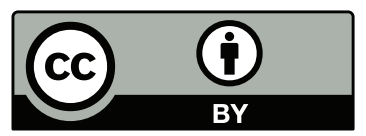

\title{
Small-scale atmospheric turbulence and its impact on laminar-to-turbulent transition on a glider airfoil
}

\author{
Amandine Guissart* \\ Technical University of Darmstadt, 64347 Griesheim, Germany \\ Jonas Romblad ${ }^{\dagger}$ \\ University of Stuttgart, 70569 Stuttgart, Germany \\ Timotheus Nemitz ${ }^{\ddagger}$ and Cameron Tropea ${ }^{\S}$ \\ Institute for Fluid Mechanics and Aerodynamics (SLA), TU Darmstadt, 64347 Griesheim, Germany
}

Using flight measurements conducted between altitudes of $700 \mathrm{~m}$ and $3000 \mathrm{~m}$, this work characterizes atmospheric turbulence and investigates the effects of an increase in turbulence level on the laminar-turbulent transition taking place on the pressure side of a laminar airfoil. Flight conditions ranging from calm to moderately turbulent and natural transition driven by Tollmien-Schlichting waves are considered. The inflow conditions are first characterized and reported using single and two-point statistics. Moreover, it is shown how characteristic parameters can be estimated from the turbulence intensity. Then, the sensitivity of the transition location to an increase of turbulence level is investigated. Flight results show a low sensitivity of the transition location to an increase of turbulence level, when the latter is not associated with significant variations of pressure gradient. Similar investigations are also conducted in a wind tunnel where the turbulence level is increased using an active grid and a significant change of the transition location is observed with increasing turbulence level. The differences in the response of the transition to freestream turbulence level in flight and in the wind tunnel is postulated to be attributable to differences in the probability density distributions of the inflow velocity fluctuations.

\section{Nomenclature}

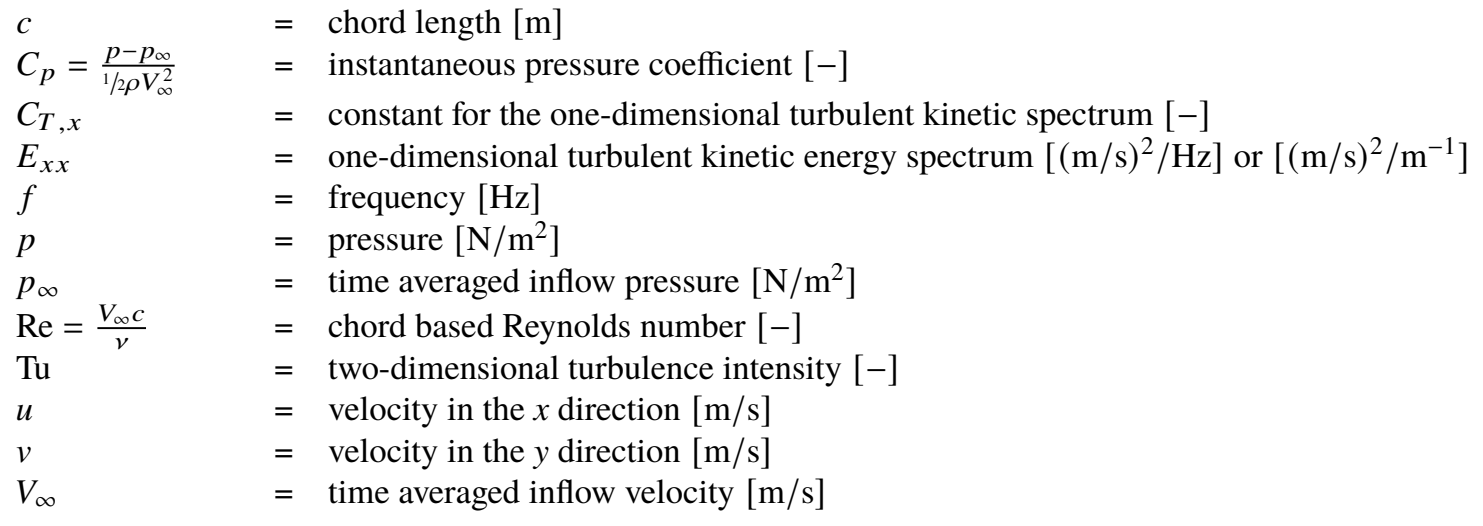

\footnotetext{
*Post-doctoral researcher, Institute of Fluid Mechanics and Aerodynamics, Flughafenstrasse 19, guissart@sla.tu-darmstadt.de.

$\dagger$ Doctoral researcher, Institute of Aerodynamics and Gas Dynamics, Pfaffenwaldring 21, jonas.romblad@iag.uni-stuttgart.de.

†Former doctoral researcher, Institute of Fluid Mechanics and Aerodynamics, Flughafenstrasse 19., inbox@ timotheus-nemitz.de.

${ }^{\S}$ Professor, Institute of Fluid Mechanics and Aerodynamics, Flughafenstrasse 19, tropea@sla.tu-darmstadt.de, AIAA member.
} 


$\begin{array}{ll}x & =\text { streamwise coordinate }[\mathrm{m}] \\ \tilde{x}=x / c & =\text { chordwise dimensionless position }[-] \\ \bar{a}=\overline{a(t)} & =\text { time or ensemble average of a quantity } a[a] \\ a(t)^{\prime}=a(t)-\bar{a} & =\text { temporal variation of a quantity } a, \text { fluctuation of } a[a] \\ \alpha & =\text { angle of attack of the airfoil }\left[{ }^{\circ}\right] \\ \delta u_{\tau}^{\prime} & =\text { velocity fluctuation increment }[\mathrm{m} / \mathrm{s}] \\ \varepsilon & =\text { turbulent energy dissipation rate }\left[\mathrm{m}^{2} / \mathrm{s}^{3}\right] \\ \kappa & =\text { wavenumber }\left[\mathrm{m}^{-1}\right] \\ v & =\text { kinematic viscosity }\left[\mathrm{m}^{2} / \mathrm{s}\right] \\ \tau & =\text { time scale used to compute } \delta u_{\tau}^{\prime}[\mathrm{s}] \\ \text { LST } & =\text { linear stability theory } \\ \text { pdf } & =\text { probability density function } \\ \text { PSD } & =\text { power spectral density } \\ \text { STD } & =\text { standard deviation } \\ \text { TS } & =\text { Tollmien-Schlichting }\end{array}$

\section{Introduction}

$T_{\text {He prediction of transition is an important prerequisite for the design of natural laminar flow (NLF) airfoils and the }}$ development of methods delaying transition to turbulent boundary layers. While the influence of pressure gradient on transition has often been studied (for instance [1,2]), the influence of inflow turbulence that can enter into the boundary layer and trigger disturbances (receptivity) is less well-known, especially for real flight conditions. The most common method of transition prediction is the $e^{n}$-method [3,4], often combined with the critical $n$-factor that depends on the turbulent intensity [5]. However, atmospheric turbulence leads to an unsteady inflow [2] and the predicted transition location is then only partially reliable. To improve the transition prediction, it is therefore important to better understand the transitional phenomena under real flight conditions. This study constitutes a first step toward this goal, as it focuses on conditions pertaining to glider flight.

Flight measurements have been conducted to investigate the laminar-turbulent transition under calm atmospheric conditions [6-10] or to study different flow control strategies (for instance [11, 12]). However, most of the time, the inflow conditions where not recorded. In particular, Zanin (1975) [6] investigated transition using a glider whose wing was instrumented with hot-wire anemometers and sublimating coating films. Inflow turbulence levels and frequency spectra were also reported. Nitsche et al. (2001) [7] studied controlled transition on an unswept motorized-glider wing using various measurement techniques. As for Seitz and Horstmann (2006) [10], they used a hot-film array to sense skin friction fluctuations provoked by Tollmien-Schlichting (TS) disturbances. They observed wave packets and reported that the breakdown to turbulence starts from within the packets with the strongest amplitudes. Various wind-tunnel investigations have also been conducted for different turbulence levels (see for instance [13, 14]). Recently, the laminar-turbulent transition was studied in flight for different levels of atmospheric turbulence [15] ranging from calm to moderately turbulent conditions (see Sec. III.A for a detailed definition of the turbulent ranges). For moderately turbulent conditions, this study identified the unsteady changes of pressure gradient caused by variations of the inflow angle (due to large scale turbulence) as one source for the modification of the transition development. However, this effect superimposes on those of increased levels of atmospheric small-scale turbulence that enter the boundary layer through receptivity and provide initial amplitudes of disturbances. Therefore, as their effects are combined, a separation between these two driving phenomena is difficult to obtain solely with flight measurements [15]. Wind tunnel and computational fluid dynamic investigations enable the separate study of these different scales, but they require a complete description of the turbulent inflow characteristics to correctly model the inflow conditions.

Atmospheric turbulent conditions have been investigated in the past from various perspectives. In particular, flight measurements investigating the atmospheric boundary layer have been conducted since the fifties [16] and since then various studies have reported, among other quantities, low order statistics such as turbulence intensities and spectra [17-24]. Among them, the most complete study has probably been conducted by Sheih et al. (1971) [20] and details energy spectra, dissipation rates and probability density distributions obtained from hot-wires installed on an aircraft and aerovanes located on a tower. More recent measurements were also conducted, aiming to further study the atmospheric conditions. However, they were either restricted to low altitude (e.g. Li et al. [25]) and/or limited in the investigated length scale range (e.g. Bodini et al. (2018) [26]). Despite this continued interest, and from the best of the authors' knowledge, a detailed characterization of the atmospheric boundary layer over a large range of length scales 
and for various turbulent conditions is not yet available for altitudes corresponding to (sailplane) flight levels. It is, therefore, difficult to define representative inflow conditions for wind tunnel or computational fluid dynamic studies.

The present study is part of a joint project that has been performed in the framework of the Luftfahrtforschungsprogramm $(\mathrm{LuFo} \mathrm{V})$ in Germany, and aims to provide a basis for further developments in transition prediction under real flight conditions. To this end, Direct Numerical Simulations [27], wind tunnel experiments [28-30] and flight experiments have been conducted to better understand the impact of atmospheric turbulence on the transition process of a laminar boundary layer. In particular, the respective effects of large and small-scale turbulence are investigated separately using numerical tools and several wind experiments. The present study summarizes findings coming from flight experiments conducted at altitudes between $700 \mathrm{~m}$ and $3000 \mathrm{~m}$. Although flight experiments have already been conducted at TU Darmstadt, inflow conditions found during the flight campaigns are reported here in detail for the first time. In particular, they are first described reporting single and two-point statistics of various orders obtained for flight conditions ranging from calm to moderately turbulent. The laminar-turbulent transition phenomenon is then described, focusing on the effects of an increase in turbulence intensity (from a calm to a lightly turbulent inflow, i.e. without unsteady changes of pressure gradient), a subject that, to the best of the authors' knowledge, has not been investigated using flight measurements. The results of these investigations are also compared to those coming from wind tunnel studies conducted by the research project partner [28-30].

\section{Methodology}

\section{A. Flight experiments: setup and measurement campaign}

\section{Experimental setup}

The flight experiments are carried out with a Grob G109b motorized glider on which several measurement devices are mounted, as depicted in Fig. 1a. The sensors enabling the study of the laminar-turbulent transition are installed in a wing glove mounted on the right wing, while the oncoming flow turbulence is characterized by hot-wires mounted on a boom attached to the glove. On a second boom mounted on the left wing, additional probes measure reference flow quantities, such as static pressure, dynamic pressure, temperature and humidity. Additionally, the angle of attack and the sideslip angle of the aircraft are measured by a wind vane (Dornier flight log) and displayed to the pilot during measurements. More information about the probes mounted on the left side can be founded in [31].

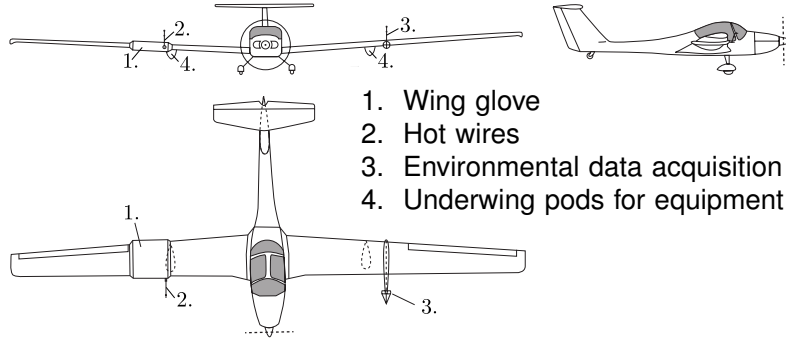

(a) Aircraft and measurement system. Reproduced from [32].

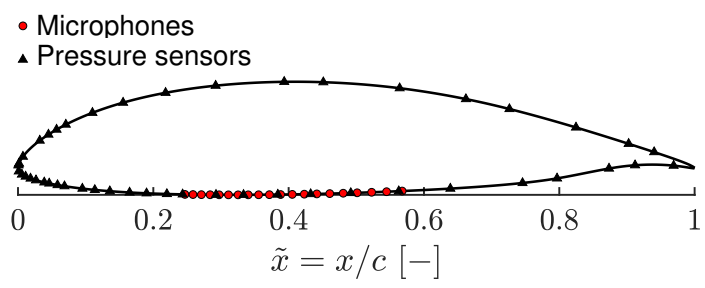

(b) Microphones and differential pressure sensors.

Fig. 1 Schematic view of the research aircraft and the measurement system and location of sensors on the wing airfoil.

The wing glove mounted on the right wing has a rectangular planform with a chord length $c$ of $1.35 \mathrm{~m}$ and a span of $1.55 \mathrm{~m}$, and is based on the laminar DU84-158 sailplane airfoil. The leading edge, the thickness and the camber of the original airfoil have been modified as described in [1] to compensate for the additional weight and drag due to the glove (airfoil geometry parameters can be found in [2]). Previous investigations showed that the cross-flow velocity on the center section of the glove is less than $2 \%$ of the freestream [1] and that the flow can be considered two-dimensional [15]. The laminar-turbulent transition process taking place along the pressure side of the airfoil is investigated with a streamwise array of 20 electret microphones (Sennheiser KE 4-211-2) mounted behind holes of $0.3 \mathrm{~mm}$ diameter. As depicted in Fig. 1b, the array is positioned from $\tilde{x}=0.25$ to 0.56 , with $\tilde{x}=x / c$, on a line located at $0.59 c$ of the fuselage side of the glove. To study the phenomenon over a spanwise direction, two rows of 16 microphones span the pressure side of glove starting at $0.51 c$ and finishing at $0.63 c$ of the fuselage side of the glove. These two spanwise rows are located at $\tilde{x}=0.31$ and $\tilde{x}=0.34$ and they are used to ensure that the studied phenomenon 
is two-dimensional (verifying that the spanwise distribution of the time averaged standard deviation of the pressure fluctuations is constant). These spanwise rows are also used to compute frequency wavenumber spectra (results not shown here but reported in [33]). In a flush-mounted configuration, the frequency response of the microphones is flat $(-3 \mathrm{~dB}$ flatness in the range [50-6000] Hz), but in the present configuration, the pinhole influences the frequency response. Therefore, the recorded signal has to be corrected and an individual calibration of each microphone is necessary. This is done with a custom calibrator designed as proposed in [34]. Finally, to prevent air leakage, the suction side of the glove was covered by a thin transparent plastic film (wrapped around the leading edge), perforated at the microphone locations.

The inflow conditions are determined by a constant temperature anemometer (see [2] for more information) using a $5 \mu \mathrm{m}$ diameter $\mathrm{X}$-wire probe (Dantec Dynamics 55P61) mounted on the right boom, extending $0.9 \mathrm{~m}$ ahead of the wing glove. The $\mathrm{X}$-wires probe measures the streamwise and vertical velocities, and thus the instantaneous angle of attack. Note that after the measuring bridge circuits, a low-pass filter with a bandwidth of $3.8 \mathrm{kHz}$ is applied to the signals, which limits the upper range of frequencies detected. The $\mathrm{X}$-wire probe is calibrated using the effective angle calibration introduced in [35] and described in detail in [15]. The anemometer outputs are not corrected for temperature changes with respect to the calibration conditions on the ground (temperature difference of around 10 to $20^{\circ} \mathrm{C}$ ), as none of the analytical correction formulas tested were able to provide satisfying precision [15]. However, the fluctuations around the time averaged inflow velocity and angle of attack can be precisely measured without correcting for ambient temperature changes (see [15] for more information on the precision retained). Therefore, the investigations discussed in the following are based on these quantities only. The flow velocity is measured using a pitot-static system, also located on the boom. A differential pressure sensor (First Sensor HCLA0025D) determines the dynamic pressure and the density of the air is obtained through environmental measurements performed on the left wing. Finally, the pressure distribution along the suction and pressure sides of the airfoil is measured with differential pressure sensors (First Sensor HCL12X5) connected to 48 pressure taps drilled into the wing glove with a diameter of $0.2 \mathrm{~mm}$. The location of the pressure sensors is visible in Fig. 1b. A static calibration of each pressure sensor is performed over the operating range with a pressure calibration device (MENSOR CPC 6000).

\section{Measurement campaigns}

The measurements were carried out between August 2018 and March 2019 from the August-Euler airfield located in Griesheim, Germany. The main goal of the flight campaigns is the characterization of the inflow conditions and the investigation of the laminar-turbulent transition for increasing turbulence level, thus, measurements are acquired over numerous flights. To avoid vibrations and electrical/acoustic noise due to the engine, data are acquired in straight gliding flight (engine shut off), from a flight level of about $3000 \mathrm{~m}$ to $700 \mathrm{~m}$. More than 20 gliding flights were conducted for conditions associated with freestream turbulent intensity $\left(\mathrm{Tu}^{20 \mathrm{~Hz}-1 \mathrm{kHz}}\right)$ ranging from below $0.01 \%$ to around $0.25 \%$. Most of the data were acquired while descending continuously from slightly above the cumulus cloud base into the convective mixed layer of the atmospheric boundary layer. Consequently, for a typical gliding flight, the turbulence intensity gradually increases for lower altitude (more information in [31]). Measurements are conducted over several intervals of $20 \mathrm{~s}$, during which the pilot attempts to match the prescribed angle of attack to the incidence angle measured by the wind vane. Calibrated airspeeds experienced during the flights range between $43 \mathrm{~m} / \mathrm{s} \mathrm{and} 54 \mathrm{~m} / \mathrm{s}$ $\left(\operatorname{Re} \in\left[3.7 \times 10^{6}, 4.3 \times 10^{6}\right]\right)$, depending on the angle of attack $\left(\alpha \in\left[-2.2^{\circ},-0.55^{\circ}\right]\right)$. Further information about the role of the pilot and the test procedure can be found in [2].

\section{B. Wind tunnel experiments}

The wind tunnel measurements were performed in the Laminar Wind Tunnel of the IAG institute at the university of Stuttgart, an open return tunnel with a base turbulence level of $\mathrm{Tu}^{10 \mathrm{~Hz}-5 \mathrm{kHz}}=0.01 \%\left(\mathrm{Tu}^{20 \mathrm{~Hz}-1 \mathrm{kHz}}=0.01 \%\right)$ in the velocity range investigated in the context of this study. Sound absorbing lining in the diffuser results in an overall sound pressure level of $76 \mathrm{dBA}$ for $40 \leq f \leq 22000 \mathrm{~Hz}$ [36]. The levels of the unweighted $1 / 3$ octave spectrum vary between $62 \mathrm{~dB}$ and $70 \mathrm{~dB}$ in the range $300 \leq f \leq 2000 \mathrm{~Hz}$ [36], where TS amplification occurs as predicted from linear stability analysis. Additional inflow turbulence is generated using an active pneumatic grid placed in the settling chamber, producing an adjustable turbulence level in the range $0.03 \% \leq \mathrm{Tu}^{10 \mathrm{~Hz}-5 \mathrm{kHz}} \leq 0.11 \%\left(0.02 \% \leq \mathrm{Tu}^{20 \mathrm{~Hz}-1 \mathrm{kHz}} \leq 0.1 \%\right)$ in the $u$-direction. With the grid, the one-dimensional turbulence intensities in $v$ and $w$-directions are larger than Tu in the $u$-direction by a factor in the order of 1.4 for $300 \leq f \leq 2000 \mathrm{~Hz}$. Freestream turbulence characteristics are measured by Constant Temperature Anemometry (CTA) using a normal probe with a $2.5 \mu$ m diameter wire. The AC part of the hot-wire signal is low-pass filtered at $15 \mathrm{kHz}$ to avoid aliasing and sampled at $44.1 \mathrm{kHz}$. The freestream velocity is set to $38 \mathrm{~m} / \mathrm{s}$ which corresponds to a Reynolds number around $\mathrm{Re}=3.4 \times 10^{6}$ (value depending on the ambient 
conditions and being slightly below the Re range investigated in flight experiments). The airfoil model, manufactured in the same molds as the wing glove for the flight measurements, is instrumented with 42 pressure taps along the chord, 21 of which are equipped with modified First Sensor HCL0050 pressure transducers capable of measuring unsteady surface pressures in the range $0 \leq f \leq 8 \mathrm{kHz}$. Boundary layer measurements were performed using CTA with a probe having a $5 \mu \mathrm{m}$ diameter wire. More information about wind tunnel experiments can be found in [28].

\section{Linear stability analysis}

Linear stability theory (LST) is used for comparison, and the results are provided by a in-house MATLAB code (see [15]). It is based on a three-step procedure: (i) the computation of the viscous pressure distribution through XFOIL 6.97, (ii) the determination of the boundary layer parameters based on a Keller-Box discretisation, and (iii) a local linear stability analysis conducted with a spectral collocation method.

\section{Post-processing of data}

To characterize the atmospheric turbulence and to investigate the laminar-turbulent transition under different inflow characteristics, the collected data have first to be post-processed. This section focuses on the post-processing related to data acquired during flight measurements. More information on the post-processing of wind tunnel data can be found in [28].

As the pressure gradient changes with angle of attack, it is of primary importance to determine precisely the local incidence angle $\alpha$ of the glove. This is achieved by visually matching the measured time averaged pressure distribution $\bar{C}_{p}(\tilde{x})$ with the results computed by the $2 \mathrm{D}$ airfoil analysis program XFOIL [37] for different values of angle of attack varying in increments of $0.05^{\circ}$. The matching procedure focuses mainly on the $\bar{C}_{p}$ distribution around the leading edge and its gradient along the stretch $x=0.05 c$ and $x=0.3 c$ (relative difference below 3\%). The measured $\bar{C}_{p}$ distribution is obtained by averaging $C_{p}=\left(p-p_{\infty}\right) / \frac{1}{2} \rho V_{\infty}^{2}$ over the $20 \mathrm{~s}$ of each measurement interval, $p-p_{\infty}$ being the pressure distribution measured by the First Sensor HCL differential pressure sensors and $V_{\infty}$ being the flow velocity measured by the pitot-static system averaged over the measurement interval.

The turbulent inflow conditions are described in terms of the following quantities. The temporal variation of angle of attack fluctuations $\alpha^{\prime}(t)$ is obtained subtracting the time averaged incidence $\bar{\alpha}$ from the time varying angle $\alpha$, i.e. $\alpha^{\prime}(t)=\alpha(t)-\bar{\alpha}$. The two-dimensional turbulence intensity Tu is computed as

$$
\mathrm{Tu}=\frac{1}{V_{\infty}} \sqrt{\frac{1}{2}\left(\overline{u^{\prime 2}}+\overline{v^{\prime 2}}\right)}
$$

To determine Tu, the fluctuations around the mean velocity $u^{\prime}=u-\bar{u}$ and $v^{\prime}=v-\bar{v}$ measured by the $\mathrm{X}$-wire probe are first filtered (band-pass filter, second-order Butterworth transfer function, cutoff frequencies $f_{1}=20 \mathrm{~Hz}$ and $f_{2}=1 \mathrm{kHz}$ ) to exclude the low frequency aircraft motion and the high frequency electronic noise. As most of the turbulent energy is contained in the low frequency fluctuations, the lower cutoff frequency has a strong impact on the computed turbulence intensity Tu and any comparison must be conducted using the same value for $f_{1}$. On the contrary, the upper cutoff frequency $f_{2}$ has a minor impact on the turbulence intensity and, in the context of this study, considering a higher value would not modify the computed Tu significantly. The one-dimensional spectrum $E_{x x}(\kappa)$ is obtained by computing the power spectral density (PSD) of the unfiltered fluctuation $u^{\prime}$ [38]. To this end, Welch's PSD estimate [39] is used, considering blocks of size $0.16 \mathrm{~s}$ with $50 \%$ overlap, and about 250 blocks. The wavenumber $\kappa=2 \pi f / V_{\infty}$ is determined using Taylor's hypothesis. The turbulent energy dissipation rate $\varepsilon$ is determined using a least square fit of $E_{x x}(\kappa)$ in the inertial subrange, within which the one-dimensional spectrum can be assumed to follow the power-law

$$
E_{x x}(\kappa)=C_{T, x} \varepsilon^{2 / 3} \kappa^{-5 / 3}
$$

where $C_{T, x}=0.49$ (Kolmogorov hypothesis). The probability density functions $\operatorname{pdf}\left(\delta u_{\tau}^{\prime}\right)$ reveals the likelihood of a velocity fluctuation increment $\delta u_{\tau}^{\prime}$, defined as $\delta u_{\tau}^{\prime}(t) \equiv u^{\prime}(t+\tau)-u^{\prime}(t)$. In this definition, $\tau$ stands for the time scale of interest and $\delta u_{\tau}^{\prime}(t)$ represents the variation in the amplitude of $u^{\prime}$ within the time $\tau$, i.e. at an effective frequency $f=1 / \tau$. It should be pointed out that the velocity fluctuation increment $\delta u_{\tau}^{\prime}(t)$ is approximately equal to the velocity increment $\delta u_{\tau}(t) \equiv u(t+\tau)-u(t)$, since $\overline{u(t)} \approx \overline{u(t+\tau)}$ (because $\tau$ is much smaller than the total time considered for averaging). To obtain $\operatorname{pdf}\left(\delta u_{\tau}^{\prime}\right)$, a histogram of $\delta u_{\tau}^{\prime}$ is first computed using 250 bins and considering only elements of $\delta u_{\tau}^{\prime}$ whose value lies within \pm 5 standard deviations (STD) around the averaged of $\delta u_{\tau}^{\prime}$. The histogram is then normalized to obtain the corresponding probability density function, i.e. the integral value is equal to unity. 
The transition process is identified using $\operatorname{STD}\left(p^{\prime}\right)$, the time varying standard deviation of the pressure fluctuations $p^{\prime}$ measured by the microphones. It is obtained dividing the signal of each microphone into segments of approximately $0.1 \mathrm{~s}$ length. The standard deviation of the pressure fluctuation is then computed for each segment, yielding a time varying STD. The frequency content of the pressure fluctuations measured at pressure taps located on the streamwise row is also studied. Again, this is done using the Welch PSD estimate and considering blocks of size $0.16 \mathrm{~s}$ with 50\% overlap.

\section{Results}

\section{A. Inflow characteristics}

Turbulence properties in the atmospheric boundary layer vary with atmospheric conditions that depend on many aspects, such as meteorological conditions, ground orographic features, diurnal changes, etc [40]. Consequently, the turbulence intensity at a particular altitude can strongly vary from one measurement to another (data acquired for different days, altitudes and locations). According to Reeh and Tropea (2015) [2], the turbulent inflow conditions can be divided into different categories based on the amplitude of the angle of attack fluctuations $\alpha^{\prime}(t)=\alpha-\bar{\alpha}$ : calm conditions, lightly turbulent conditions, moderately turbulent conditions and turbulent conditions. Several temporal variations associated with the different categories experienced in flight are reported in Fig. 2a. For calm conditions $\left(\mathrm{Tu}^{20 \mathrm{~Hz}-1 \mathrm{kHz}}<0.02 \%\right)$, the angle of attack is nearly constant and only low frequency fluctuations associated with the plane motion are visible. As the turbulence level increases, the inflow is said to be first lightly turbulent, for $\alpha^{\prime}<1^{\circ}$ $\left(0.02 \%<\mathrm{Tu}^{20 \mathrm{~Hz}-1 \mathrm{kHz}}<0.15 \%\right)$, and then moderately turbulent for $1^{\circ}<\alpha^{\prime}<3^{\circ}\left(0.15 \%<\mathrm{Tu}^{20 \mathrm{~Hz}-1 \mathrm{kHz}}<0.24 \%\right)$. Higher fluctuations of the incidence angle corresponding to a turbulent inflow were only rarely experienced during this study (as shown in Fig. 3a). The one-dimensional turbulent kinetic energy spectra $E_{x x}(f)$ that correspond to the temporal evolution of $\alpha^{\prime}(t)$ depicted in Fig. 2a are shown in Fig. 2b (colored plain lines). The spectral model proposed by Pope (2000) [38] is also depicted (dashed black lines), and matches well the experimental results in the inertial and the dissipation ranges. For each turbulence intensity, $E_{x x}(f)$ clearly exhibits the Kolmogorov $-5 / 3$ power-law decay [41] within a frequency range corresponding to the inertial subrange [38]. For higher frequencies, the spectra exhibit the typical decrease corresponding to the dissipation range. The latter is however not fully resolved and below a certain energy level (at which the spectra starts to level off), only electrical noise is recorded. Finally, the several peaks visible around $400 \mathrm{~Hz}$ and $2000 \mathrm{~Hz}$ are a result of electromagnetic disturbances [15]. Because of the acquisition time limited to $20 \mathrm{~s}$, the spectra are truncated in the low frequency range corresponding to the energy-containing scales (large scales [38]). Finally, Fig. 2b shows also the increase of energy at all time scales, corresponding to an increase in turbulence level as the spectra are shifted upwards ( $\varepsilon$ is representative of the power density in the inertial subrange (see Eq. 2)).

Figure 2 shows the effects of an increase in turbulence level considering only some representative samples. However, as the flight measurement campaign led to more than 550 measurement samples, a statistical analysis provides better insight into the main characteristics of atmospheric turbulence. Results of this analysis are depicted in Fig. 3. In particular, the turbulent conditions are investigated in terms of: (i) the connection between the turbulent energy dissipation rate $\varepsilon$ and the turbulence intensity $\mathrm{Tu}^{20 \mathrm{~Hz}-1 \mathrm{kHz}}$, (ii) the turbulent energy spectra $E_{x x}(f)$, (iii) the probability density functions $\operatorname{pdf}\left(\delta u_{\tau}^{\prime}\right)$ of the velocity fluctuation increments $\delta u_{\tau}^{\prime}$ for various time scales $\tau$, and, finally, (iv) the corresponding standard deviations.

The black dots in Fig. 3a represent the value pairs $(\mathrm{Tu}, \varepsilon)$ obtained from each available measurement sample. It can be seen that most of the measurements were conducted for calm to lightly turbulent conditions, while a few data points were also collected for moderately turbulent ones. For calm conditions, the turbulent energy dissipation rate $\varepsilon$ increases rapidly with the turbulence intensity $\mathrm{Tu}^{20 \mathrm{~Hz}-1 \mathrm{kHz}}$, as $\varepsilon$ is 1000 times higher for $\mathrm{Tu}^{20 \mathrm{~Hz}-1 \mathrm{kHz}}=0.01 \%$ than for $\mathrm{Tu}^{20 \mathrm{~Hz}-1 \mathrm{kHz}}=0.03 \%$. For lightly to moderately turbulent conditions, the dissipation rate $\varepsilon$ continues to increase with Tu but less rapidly. As shown by the agreement between the black dots and the red line in Fig. 3a, the increase in turbulent energy dissipation rate with the turbulence intensity can be determined using $\varepsilon=\beta V_{\infty}^{3} \mathrm{Tu}^{3}$, where $\beta^{-\frac{2}{3}}=-\frac{3}{2} C_{T, x}\left(\frac{2 \pi}{V_{\infty}}\right)^{-2 / 3}\left[f_{2}^{-2 / 3}-f_{1}^{-2 / 3}\right], f_{1}$ and $f_{2}$ being respectively the lowest and the highest frequencies of the band-pass filter used to compute the turbulence intensity (see Sec. II.D). In other words, and as showed by Chen (1974) [42], this means that the energy dissipation rate can be inferred from the Kolmogorov energy spectrum (see Eq. 2). In the following, several samples are combined to further characterize the changes in atmospheric conditions as the turbulence level increases. To do so, several Tu ranges are selected and the results obtained for the data samples in 


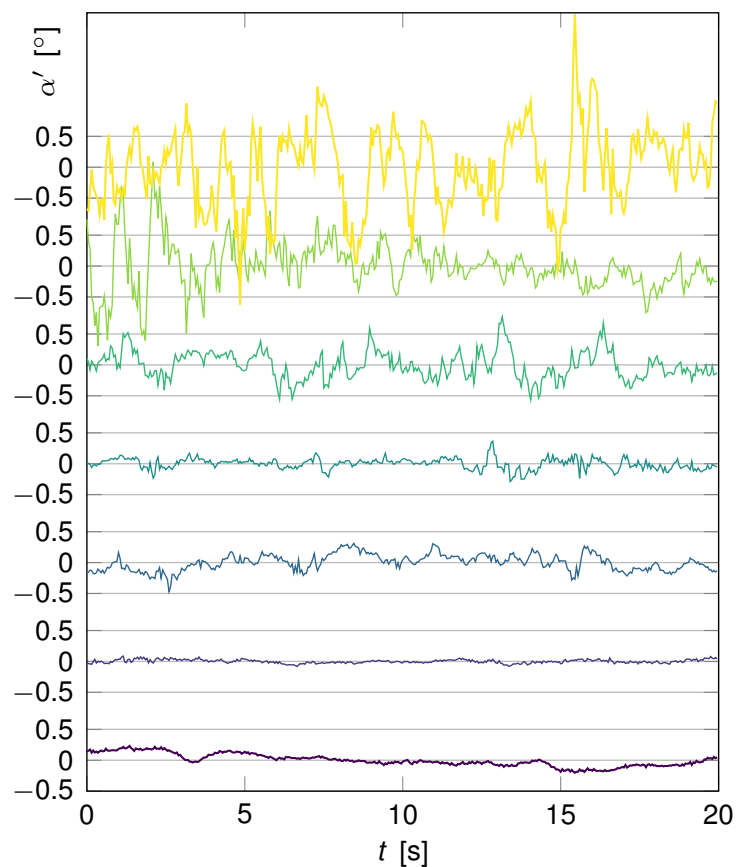

(a) Temporal variation of the unfiltered angle of attack fluctuation $\alpha^{\prime}(t)$.

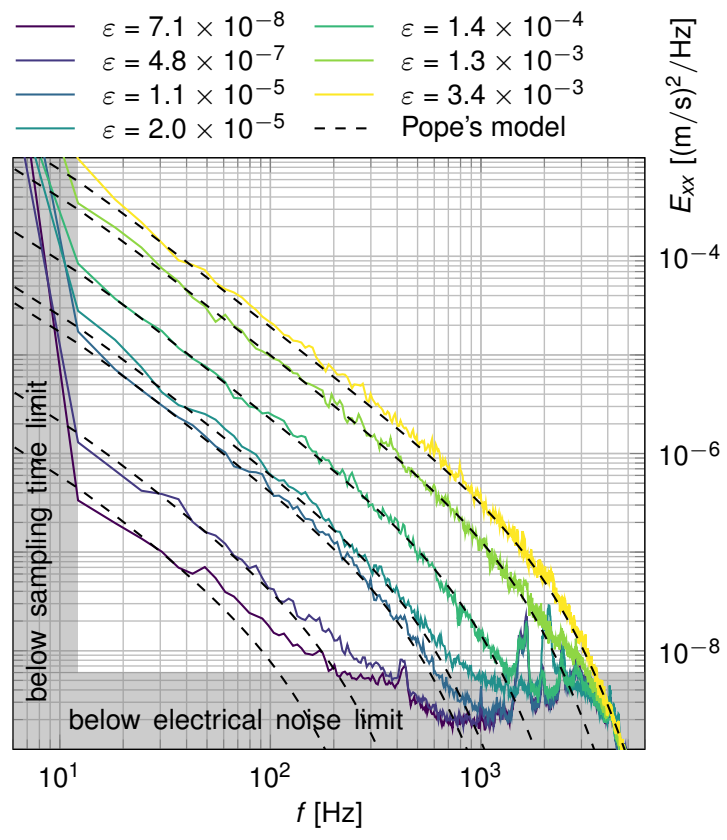

(b) One-dimensional turbulent kinetic energy spectrum $E_{x x}(f)$.

Fig. 2 Characteristics of the inflow turbulence for different Tu corresponding to different flight experiments.

each range are ensemble averaged. The different Tu ranges considered are designated by shaded colored areas in Fig. 3a.

Figure $3 \mathrm{~b}$ shows (colored lines) the one-dimensional turbulent kinetic energy spectra $E_{x x}(f)$ obtained from this ensemble averaging procedure, retaining samples only if the are not more than 2.5 standard deviations from the mean. In addition, the comments relative to the features of $E_{x x}(f)$ made in light of Fig. $2 \mathrm{~b}$ still apply here. Figure $3 \mathrm{~b}$ also shows how the level of energy contained in the different frequency ranges varies with turbulence intensity. As the turbulent level increases, the energy increases less rapidly. As the dissipation rate $\varepsilon$ is an image of $E_{x x}$ in the inertial subrange, this behavior results from the variation $\varepsilon(\mathrm{Tu})$ depicted in Fig. 3a. This means that the energy contained in the different time scales of the atmospheric boundary layer can be estimated a priori for a particular value of Tu. More precisely, the inertial subrange and the dissipation range of the turbulent kinetic energy spectrum $E_{x x}(f)$ can be modeled using the spectrum model proposed by Pope (2000) [38] and a relation linking the dissipation rate $\varepsilon$ to the turbulence level Tu [42]. The resulting spectrum can then be used as an input for simulations or wind tunnel experiments.

Although the one-dimensional turbulent energy spectrum $E_{x x}(f)$ highlights how the energy is distributed over frequency, it does not describe how the velocity fluctuations are distributed in terms of amplitude, i.e. if a particular level of energy is achieved because of a few large amplitude fluctuations or many small amplitude oscillations. To provide a more complete description of the atmospheric turbulence, probability density functions are computed from the time varying velocity fluctuation $u^{\prime}(t)$. More precisely, the probability density distribution of the velocity fluctuation increments $\delta u_{\tau}^{\prime}(t, \tau) \equiv u^{\prime}(t+\tau)-u^{\prime}(t)$ is of interest here. As $\delta u_{\tau}^{\prime}$ represents the amplitude of an increment of velocity fluctuation for a particular time scale $\tau$ (or frequency $1 / \tau$ ), the corresponding probability density function $\operatorname{pdf}\left(\delta u_{\tau}^{\prime}\right.$ ) highlights the likelihood for an increment $\delta u_{\tau}^{\prime}$ of a particular amplitude to occur.

Figure $3 \mathrm{c}$ depicts the probability density functions of $\delta u_{\tau}^{\prime} / \operatorname{STD}\left(\delta u_{\tau}^{\prime}\right)$, i.e. the velocity fluctuation increment normalized by its standard deviation. The different pdfs are obtained for time scales corresponding to $1 / \tau=100 \mathrm{~Hz}$, $250 \mathrm{~Hz}, 500 \mathrm{~Hz}$ and $1000 \mathrm{~Hz}$, which are marked by dashed lines in Fig. 3b. Similar to the spectra in Fig. 3b, results are provided for turbulence levels from an ensemble averaging procedure that uses samples lying in the different Tu ranges visible in Fig. 3a. Samples are retained only if their corresponding pdf is not more than 3 standard deviations from the mean. The colored lines in Fig. 3c show the pdfs (ensemble-averages) obtained for several turbulence levels (represented by the dissipation rate $\varepsilon$ ) and time scales $\tau$. The pdfs that correspond to energy levels below the electrical noise limit (see Fig. 3b) are not shown. For comparison purposes, the figure also depicts (black lines) the distribution corresponding to the normal distribution with a mean equal to zero and standard deviation equal to one. Looking at the pdfs obtained for $1 / \tau=100 \mathrm{~Hz}$ (Fig. 3c, upper left), it can be seen that the "peakedness" of the pdfs obtained from flight measurement is 


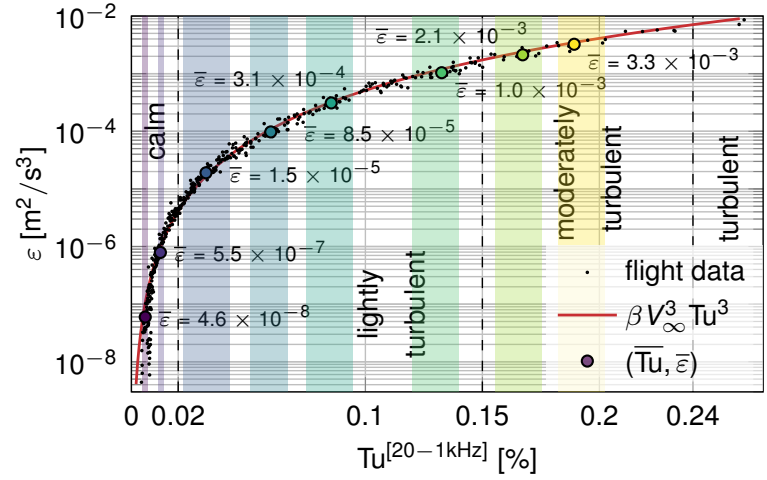

(a)
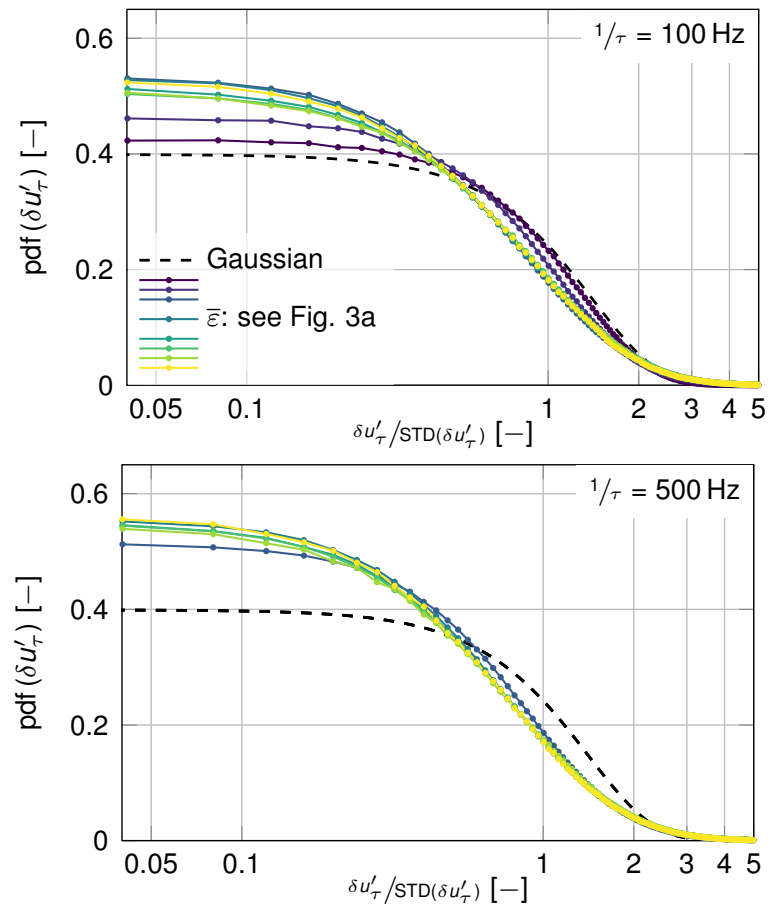

(c)

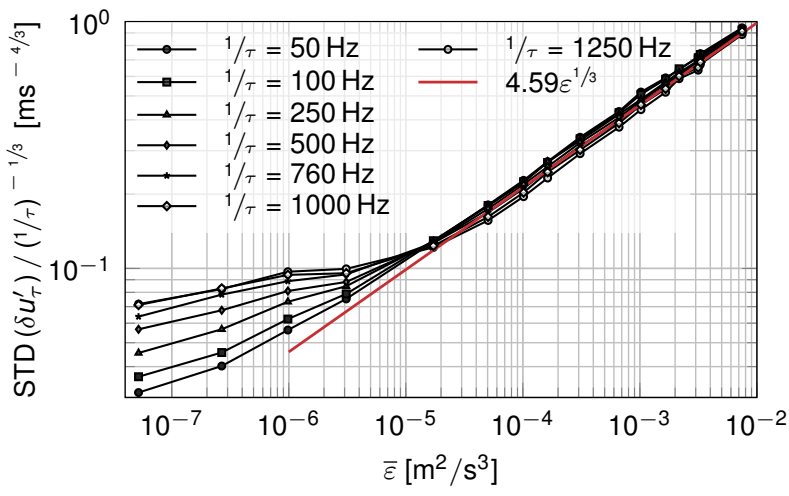

(d)

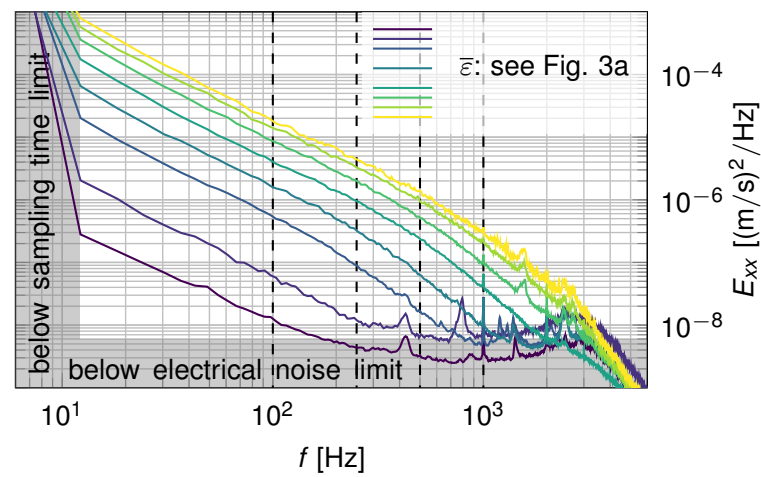

(b)
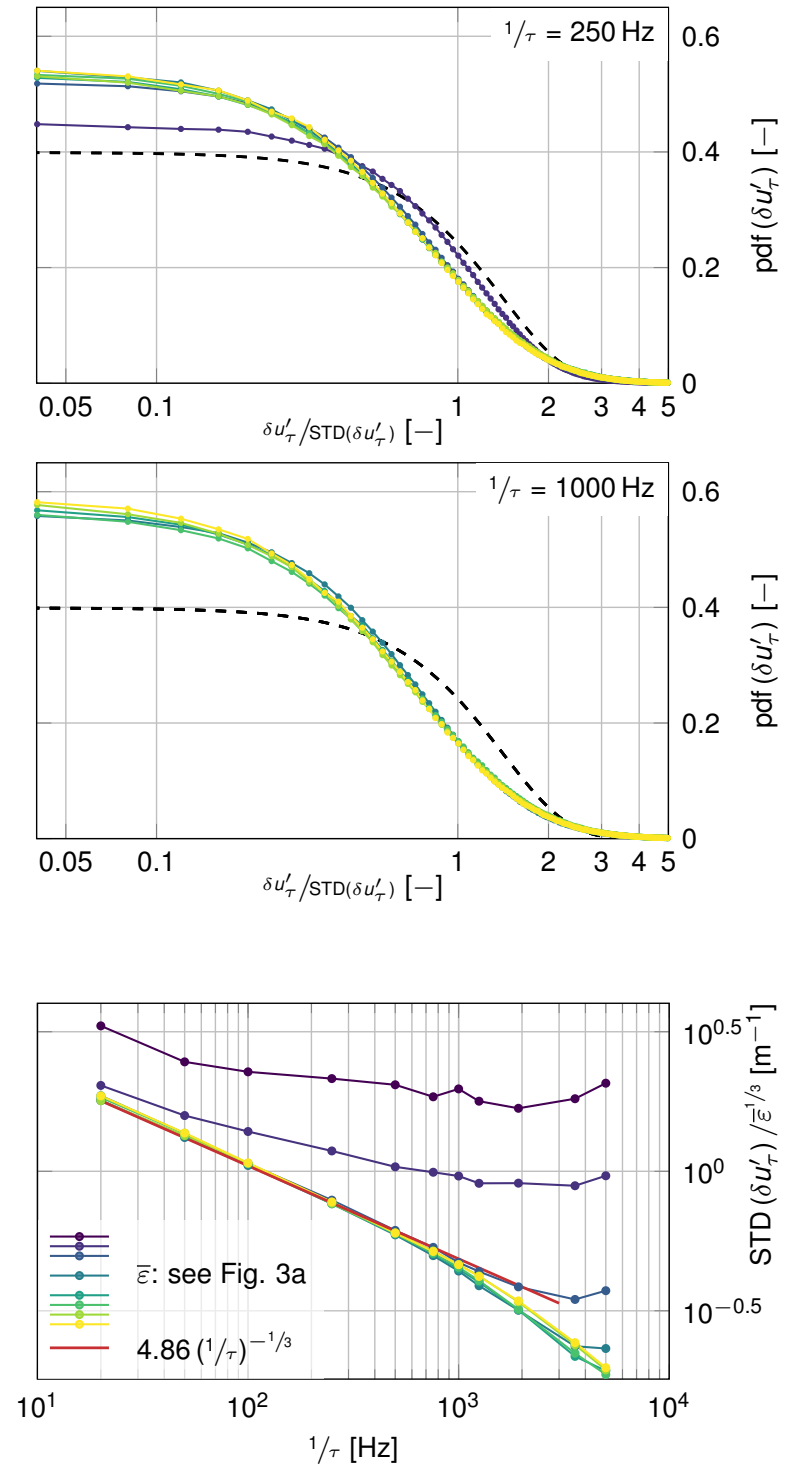

(e)

Fig. 3 Characteristics of the inflow turbulence based on ensemble averaged data for different Tu ranges. (a): Turbulent energy dissipation rate as a function of the turbulence intensity. (b): Ensemble averaged one-dimensional turbulent kinetic energy spectrum $E_{x x}(f)$. (c): Ensemble averaged pdf of the velocity fluctuation increment $\delta u_{\tau}^{\prime}$. (d) and (e): Ensemble averaged STD of the velocity fluctuation increment $\delta u_{\tau}^{\prime}$. Ensemble averages depicted in (b) to (e) are computed from the data contained in the ranges shaded in (a). 
higher than those corresponding to the normal distribution. In other words, the events with a low $\left(\delta u_{\tau}^{\prime} \lesssim 0.5 \operatorname{STD}\left(\delta u_{\tau}^{\prime}\right)\right)$ and intermediate $\left(0.5 \mathrm{STD}\left(\delta u_{\tau}^{\prime}\right) \lesssim \delta u_{\tau}^{\prime} \lesssim 2 \mathrm{STD}\left(\delta u_{\tau}^{\prime}\right)\right)$ increment of amplitude are respectively more and less likely to occur than if the probability density function would have been normally distributed. This corresponds also to a greater extremity of outliers $\left(\delta u_{\tau}^{\prime} \gtrsim 2 \operatorname{STD}\left(\delta u_{\tau}^{\prime}\right)\right)$ compared to the normal distribution (not clearly visible in Fig. 3c because of the linear $y$-axis), but the probability associated with such high amplitude excursions is fairly small (lower than 0.04). This has also been highlighted by Sheih et al. (1971) [20] who reported more heavy-tailed pdfs compared to the Gaussian distribution and peak values (value of $\operatorname{pdf}(0)$ ) around 0.5 , i.e. higher than the value corresponding to the Gaussian function (results corresponding to $\varepsilon=6 \times 10^{-4} \mathrm{~m}^{2} / \mathrm{s}^{3}$ and $1 / \tau=1800 \mathrm{~Hz}$ ). Comparing the results for different dissipation rates $\varepsilon$, it appears that the "peakedness" of the pdfs corresponding to calm conditions is lower than for more turbulent inflow conditions, the pdf distribution becoming closer to a Gaussian function as $\varepsilon$ decreases. Additionally, it can be seen that an increase of $1 / \tau$ to $250 \mathrm{~Hz}, 500 \mathrm{~Hz}$ and $1 \mathrm{kHz}$ correlates with an increase of the "peakedness".

To fully characterize the probability density function of the velocity fluctuation increments $\delta u_{\tau}^{\prime}$, the standard deviation corresponding to each pdf has to be analyzed. Figures $3 \mathrm{~d}$ and $3 \mathrm{e}$ show how $\operatorname{STD}\left(\delta u_{\tau}^{\prime}\right)$ varies with the dissipation rate $\varepsilon$ (i.e. the turbulence level) and the time scale $\tau$. In particular, a dependency in $\varepsilon^{1 / 3}$ and $(1 / \tau)^{-1 / 3}$ is visible for inflow conditions corresponding to lightly to moderately turbulent conditions $\left(\varepsilon \gtrsim 2 \times 10^{-5} \mathrm{~m}^{2} \mathrm{~s}^{-3}\right)$ and time scales leading to $1 / \tau \lesssim 800 \mathrm{~Hz}$, which mostly correspond to values $(\varepsilon, 1 / \tau)$ leading to a point lying in the inertial subrange of the turbulence spectrum (see Fig. 3b). Therefore, in the inertial range, the standard deviation of the velocity fluctuation increments can be approximated as $\operatorname{STD}\left(\delta u_{\tau}^{\prime}\right) \propto \varepsilon^{1 / 3}$ and $(1 / \tau)^{-1 / 3}$. The proportionality appears to be a function of the freestream velocity $V_{\infty}$. Using Taylor's hypothesis to rescale the frequency $1 / \tau$ as a function of the wavenumber $\kappa$, $\operatorname{STD}\left(\delta u_{\tau}^{\prime}\right)$ can be approximated by $\operatorname{STD}\left(\delta u_{\tau}^{\prime}\right) \approx 2.4 \varepsilon^{1 / 3} \kappa^{-1 / 3}$, if the considered wavenumber belongs to the inertial subrange. This functional form valid in the inertial range corresponds to the one derived by dimensional analysis from the Kolmogorov's hypotheses (see [43]). In conclusion, as for the energy content in the different time scale of the atmospheric boundary layer, the pdfs presented in Figs. 3c to $3 \mathrm{e}$ enable a priori modelling of the velocity fluctuations for different turbulence levels.

\section{B. Laminar-turbulent transition and inflow turbulence}

This section first shows that, for calm inflow conditions, the transition phenomenon measured on the pressure side of the airfoil exhibits characteristics of natural transition driven by Tollmien-Schlichting waves. Then, the sensitivity of the transition location to an increase in turbulence level is investigated using both flight and wind tunnel results. Finally, discrepancies between flight and wind tunnel inflow are highlighted and discussed.

\section{Calm inflow conditions}

This section presents results obtained for flight conditions corresponding to a calm inflow $\left(\mathrm{Tu}^{20 \mathrm{~Hz}-1 \mathrm{kHz}}<0.01 \%\right)$. The frequency content of the surface pressure along the chord of the airfoil is first presented and compared to wind tunnel and linear stability theory results. Then, the transition development is analyzed through disturbance amplification rates and the discrepancies with the results from the linear stability theory are discussed. The data obtained for calm flight conditions are analyzed further in $[28,33]$.

Spectra of chordwise signals Figure 4 depicts the PSD of the pressure fluctuations $p^{\prime}$ measured at taps lying chordwise on the pressure side of the airfoil for results obtained from experiments conducted at two slightly different combinations $(\alpha, \mathrm{Re})$, one corresponding to the flight conditions, i.e. $(\alpha, \mathrm{Re})^{\text {flight }}=\left(-1.35^{\circ}, 3.6 \times 10^{6}\right)$, the other to the wind tunnel experiments, i.e. $(\alpha, \mathrm{Re})^{\mathrm{WT}}=\left(-1.4^{\circ}, 3.4 \times 10^{6}\right)$. Both Figs. $4 \mathrm{a}$ and $4 \mathrm{~b}$ exhibit the characteristic downstream variation of spectra corresponding to a TS-waves driven laminar-turbulent transition process. For pressure taps located most upstream (lowest $\tilde{x}$ ), the flow is fully laminar. Therefore, the amplitude of $p^{\prime}$ is low and the corresponding PSD level is even below the electronic noise floor. The PSD spectra corresponding to pressure taps associated with larger $\tilde{x}$ (more downstream) depict the typical hump associated with TS-waves. From linear stability theory (LST), the frequencies associated with the most amplified disturbances in the stretch $\tilde{x} \in[0.3,0.5]$ lie in the range $[900,1200] \mathrm{Hz}$ and $[800,1100] \mathrm{Hz}$ for flight and wind tunnel measurements, respectively (slightly higher Re for the former case). This is in accordance with the frequencies of the TS-hump. This TS-hump is further amplified and broadens as $\tilde{x}$ increases, showing a shift of its maximum toward lower frequencies. Before reaching its highest amplitude, the TS-hump exhibits higher harmonics. Then, the spectra corresponding to the maximum amplitude show an increase at frequencies both below and above the most amplified frequencies. This increase corresponds to non-linear effects taking place at the end of the transition process. Finally, as the boundary layer becomes fully turbulent, the spectra corresponding to the most 
downstream pressure taps no longer exhibit amplification at any particular frequency. When rescaled to remove the effects of the velocity $V_{\infty}$ and kinematic viscosity $v$ (appearing in the chord based Reynolds number $\operatorname{Re}=V_{\infty} c / v$ ), the spectra in Fig. 4a compare quantitatively fairly well with those in Fig. 4b. This rescaling is done by non-dimensionalizing $f$ as $F=2 \pi f v / V_{\infty}^{2}$ and the PSD spectra have then to be analyzed looking at the top abscissa of Fig. 4a and Fig. 4b. Finally, the transition occurs slightly more upstream in flight than in the wind tunnel, as the largest amplitude spectra is measured at a pressure tap located at $\tilde{x}=0.42$ and $\tilde{x}=0.46$ in Figs. 4a and 4b, respectively. This discrepancy is probably due to a small difference in the airfoil profile (see Fig. 5 and the corresponding discussion).

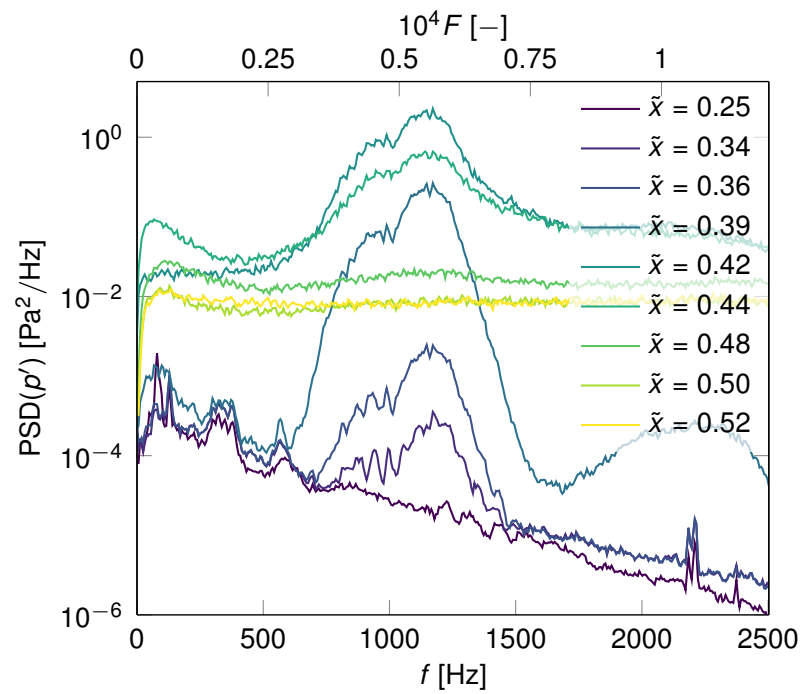

(a) Flight results.

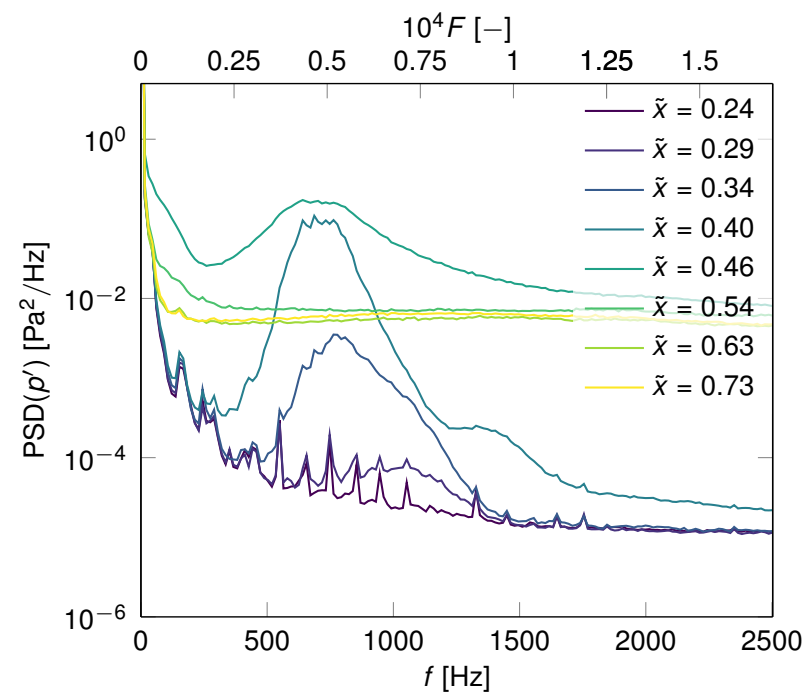

(b) Wind tunnel results.

Fig. 4 PSD of time varying surface pressure measured along the chord of the airfoil pressure side.

Amplification of disturbances Figure 5 depicts the chordwise growth of disturbances inside the boundary layer for calm conditions, both for flight and wind tunnel measurements. These results are also compared to those from a LST computation (plain lines in Fig. 5). In particular, the resulting amplification rates $\ln A / A_{0}$ are depicted for the two combinations $(\alpha, \mathrm{Re})$ corresponding to flight and wind tunnel. The time averaged standard deviation of the pressure and the velocity fluctuations, i.e. $\overline{\operatorname{STD}\left(p^{\prime}\right)}$ and $\overline{\operatorname{STD}\left(u^{\prime}\right)}$, are reported for the flight and wind tunnel experiments, respectively. To compare with LST results, a pass-band filter $( \pm 5 \%)$ around the main frequency of the TS-waves is applied on the measured fluctuations, this main frequency corresponding to $908 \mathrm{~Hz}$ and $1150 \mathrm{~Hz}$, respectively for the wind tunnel and the flight results. Moreover, for comparison purposes, the experimental results are shifted vertically, adjusting the initial disturbance level $A_{0}^{\star}$ (true value unknown) such that the second measurement point in the amplification region matches the corresponding LST results (as an example, a similar procedure was followed in [44]). Looking at flight results, a first plateau $(\tilde{x}<0.35)$ that corresponds to the electrical noise measured, where the pressure fluctuations are very weak (laminar region and beginning of amplification region), is followed by a rapid increase and then a peak of $\overline{\operatorname{STD}\left(p^{\prime}\right)}$ (amplification region, $\tilde{x} \in[0.35-0.43]$ ). Then $\overline{\operatorname{STD}\left(p^{\prime}\right)}$ decreases to finally form a second plateau (turbulent region $\tilde{x}>0.43$ ). For the wind tunnel case, only the results corresponding to measurements of near wall maxima in the amplification region are reported (more information in [28]). It can be seen that the chordwise growth of amplification measured in the wind tunnel match well with the LST results. However, the chordwise growth measured in flight is much higher than the one predicted by LST. This discrepancy is probably due to a slight difference between the prescribed and the final shape of the glove. Comparing the glove airfoil to a metallic negative template of the prescribed airfoil, it appeared that the mounting of microphones led to a change in the structural rigidity of the material, causing in turn a slight modification of the airfoil on the pressure side (maximum $1.5 \mathrm{~mm}$ ) for $\tilde{x} \in[0.2,0.5]$. The chordwise distribution of discrepancies was estimated using the negative template, and LST was applied to the obtained modified airfoil. The dashed line in Fig. 5 depicts the amplification rates obtained from these investigations and shows that the modified shape is associated with a faster increase of the amplification rates for $\tilde{x}>0.3$. This leads to a better match of the flight results with those from LST in the second part of the amplification region $(\tilde{x} \in[0.37-0.41])$. Moreover, comparing the value 
of $\tilde{x}$ obtained at a same disturbance level for the dashed and the plain black lines in Fig. 5, it appears that the modified airfoil leads to a transition occurring more upstream, which explains the discrepancy pointed out in Sec. III.B.1. Further investigations reported in [33] confirmed that the discrepancies with LST and wind tunnel results can be explained by a small difference in the investigated airfoil.

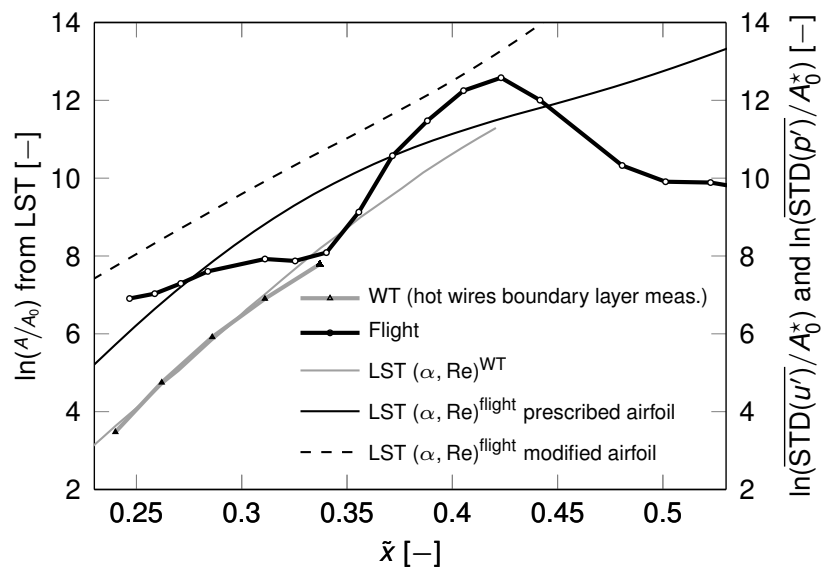

Fig. 5 Time averaged development of the laminar-turbulent transition obtained from flight and wind tunnel experiments and comparison with LST results.

In conclusion, the phenomena measured in flight and in the wind tunnel exhibit the features typical of the TS-waves driven transition (this is further detailed in $[28,33]$ ). The setup is thus appropriate to study the impact of an increase in the inflow turbulence level. In particular, the effects of small-scale turbulence are studied in the next section.

\section{Increasing turbulence level}

The effects of an increase in the inflow turbulence intensity are reported here from : (i) flight measurements under different atmospheric conditions, and (ii) wind tunnel measurements using an active grid for increasing turbulence levels [28]. Figure 6 depicts the chordwise growth of disturbances obtained from the two approaches at the respective combination $(\alpha, \operatorname{Re})$ specified above. In particular, flight results are depicted in Figs. 6a to 6d, which represent the time varying $\operatorname{STD}\left(p^{\prime}\right)$ for calm to lightly turbulent inflow conditions (shown in Fig. 2), and in Fig. 6e, which shows the corresponding chordwise evolution of $\overline{\operatorname{STD}\left(p^{\prime}\right)}$. For calm conditions, the transition location $\tilde{x}^{t r}$ remains relatively constant in time, as depicted in Fig. 6a. For increasing turbulent levels (Figs. 6b to 6d), a time variation in of the transition location (defined as the peak of $\operatorname{STD}\left(p^{\prime}\right)$ ) is visible, but the time averaged distribution is still very similar to that obtained for calm conditions. This is confirmed by the time averaged distribution of $\operatorname{STD}\left(p^{\prime}\right)$ in Fig. 6e, that shows no significant change in the transition location in the range $4.8 \times 10^{-7} \mathrm{~m}^{2} \mathrm{~s}^{-3} \leq \varepsilon \leq 1.4 \times 10^{-4} \mathrm{~m}^{2} \mathrm{~s}^{-3}$, while for $\varepsilon=1.3 \times 10^{-3} \mathrm{~m}^{2} \mathrm{~s}^{-3}$ a small upstream shift is seen. The temporal variations of $\tilde{x}^{t r}$ visible in Fig. $6 \mathrm{~d}$ correlated with the large fluctuations of incidence angle shown in Fig. $2 \mathrm{a}$ and are therefore due to a modification of the pressure gradient (see Sec. III.B.1) rather than solely to an increase of the turbulence intensity.

The chordwise distributions of $\overline{\operatorname{STD}\left(p^{\prime}\right)}$ obtained in wind tunnel for several values of the turbulent energy dissipation rate are depicted in Fig. 6f. Compared to the flight results (Fig. 6e), a significant variation of the transition location with the turbulence level can be observed. Moreover, an increase in small-scale turbulence in the wind tunnel also leads to an increase in frequency of the amplified TS waves while the transition process changes gradually from a quasi-uniform nature to a structure with formation of isolated wave packets which grow into turbulent spots (see [28]). These modifications are not observed for flight results. The reasons for these discrepancies remain unknown. However, the following section will show that differences in inflow between the wind tunnel and flight exist and might lead to discrepancies in the laminar-turbulent transition.

\section{Comparison of flight and wind tunnel inflow characteristics}

The flight and wind tunnel turbulent conditions can be compared analyzing Figs. 7 and 8, where colored plain lines stand for flight results and colored dashed lines with triangle marks correspond to wind tunnel results. The dashed 
black lines stand for the Pope's model for the energy spectrum corresponding to $\varepsilon=7.1 \times 10^{-8} \mathrm{~m}^{2} / \mathrm{s}^{3}$ (highest energy level) and $\varepsilon=5 \times 10^{-8} \mathrm{~m}^{2} / \mathrm{s}^{3}$ (lowest energy level). The same color represents a similar level of turbulence for both the flight and the wind tunnel inflows, which means, in the context of this study, a similar turbulent energy dissipation rate $\varepsilon$. For flight results, the dissipation rate is obtained by fitting the Kolmogorov power-law (Eq. (2)) to the data in the inertial subrange, while, for wind tunnel results, the dissipation rate $\varepsilon$ is determined by matching Pope's model [38] in the dissipation range. This approach is preferred to the direct comparison of the turbulent intensity value, because the lack of energy in the inertial range visible for the wind tunnel data (see Fig. 7) would have led to an artificially reduced value of turbulent intensity Tu.

Regarding the spectra depicted in Fig. 7, major discrepancies are visible in the energy content toward lower frequencies. Because of the finite length scales afforded by the wind tunnel confinement, the active grid used to generate turbulence fails to reproduce the energy level in the atmosphere for large scales and lower frequencies of the inertial subrange. Nonetheless, for the highest frequencies and in the dissipative subrange, a fair match of the energy content is visible, both when comparing wind tunnel and the flight results, or measurements and Pope's model. The discrepancies
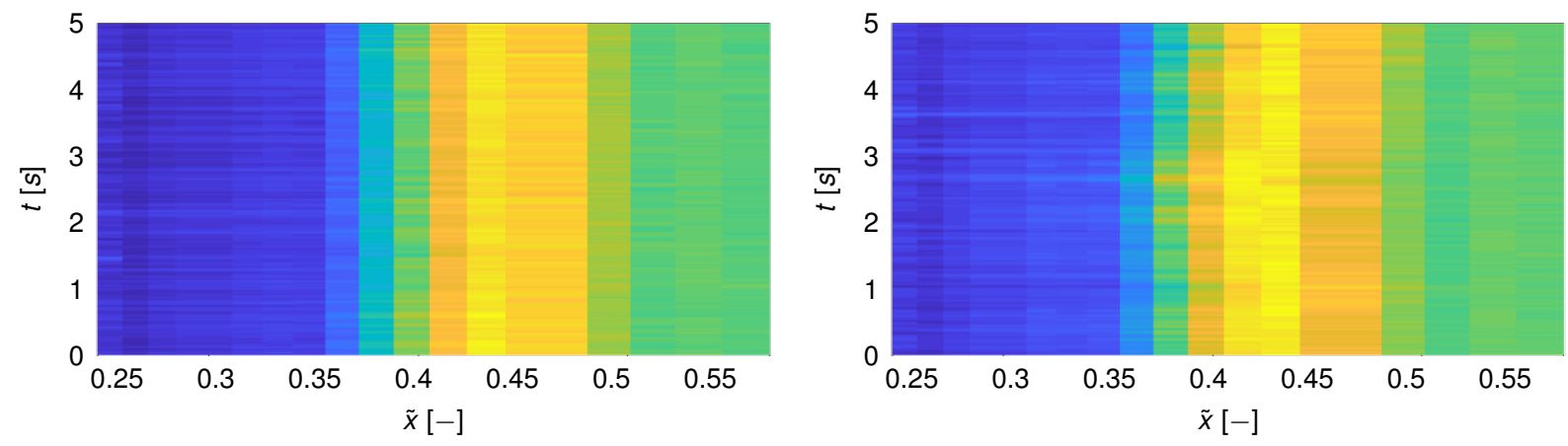

(a) $\varepsilon=4.8 \times 10^{-7} \mathrm{~m}^{2} \mathrm{~s}^{-3}$

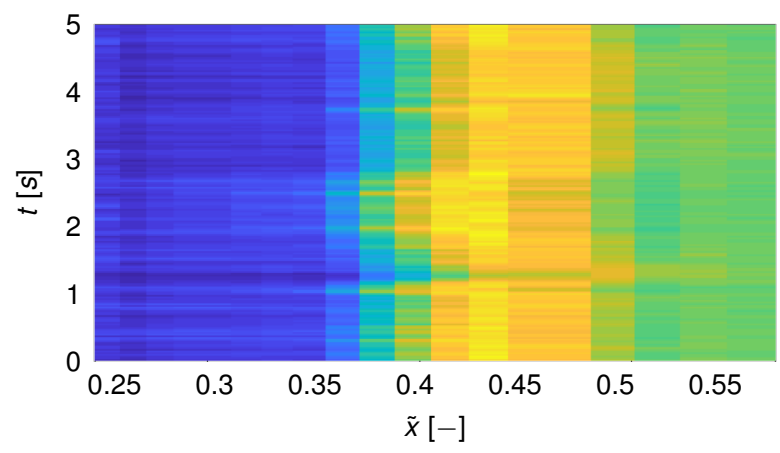

(b) $\varepsilon=1.1 \times 10^{-5} \mathrm{~m}^{2} \mathrm{~s}^{-3}$

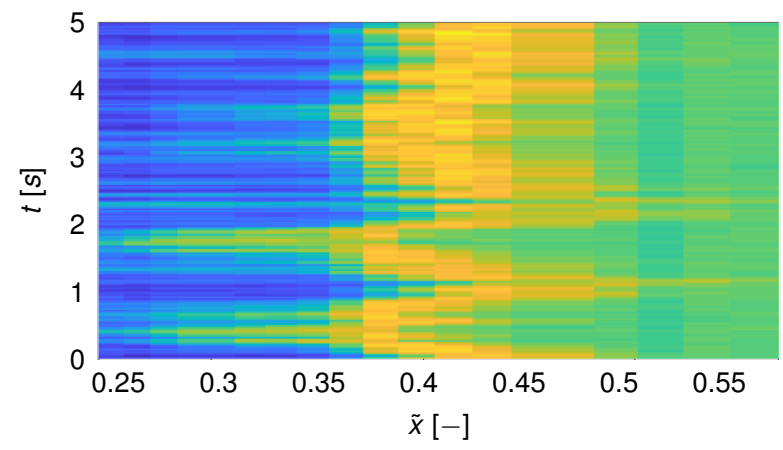

(c) $\varepsilon=1.4 \times 10^{-4} \mathrm{~m}^{2} \mathrm{~s}^{-3}$.

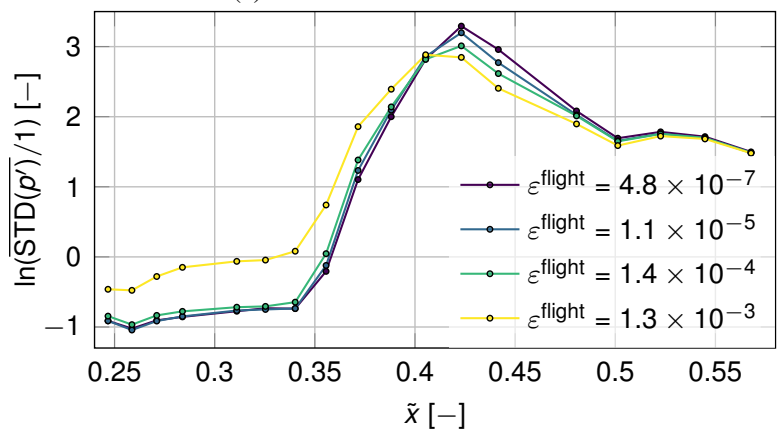

(e) Flight results.

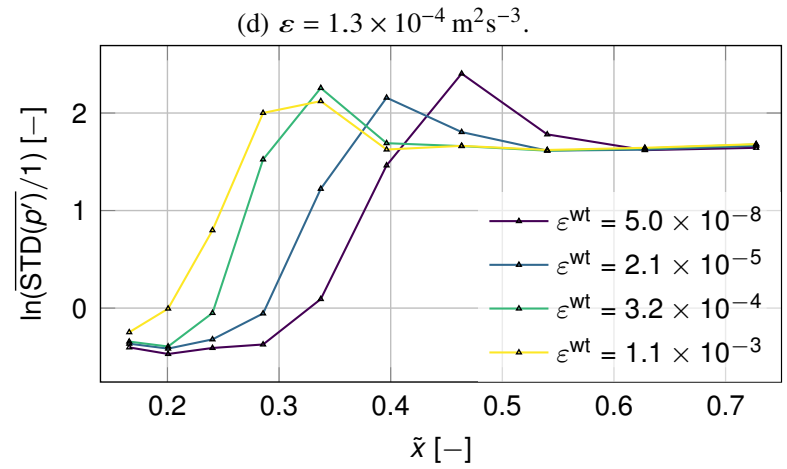

(f) Wind tunnel results.

Fig. 6 Time varying (a-d) and time averaged (e) developments of the laminar-turbulent transition for calm to lightly turbulent flight conditions. Time averaged developments measured in wind tunnel for similar ranges of turbulent energy dissipation (f). 
observed for low energy levels are assumed to be due to electrical noise. A level of turbulent energy comparable to the atmospheric one is obtained in the range of frequencies where the TS-amplification is supposed to occur in the context of the present studies (gray area). Because here TS-waves are responsible for the transition, it can be argued that an increase of the artificially generated turbulence in the wind tunnel should lead to effects on transition comparable to an increase in small-scale atmospheric turbulence [28].

Figure 8 compares the probability density distributions obtained from the flight and the wind tunnel inflows, for $1 / \tau=1000 \mathrm{~Hz}$ and $1 / \tau=750 \mathrm{~Hz}$ (frequencies corresponding to the respective TS-peak in the pressure fluctuations spectra $\left.\operatorname{PSD}\left(p^{\prime}\right)\right)$. The colored plain lines and dashed lines with triangle marks correspond to the flight and wind tunnel measurements, respectively. The dashed black line stands for a Gaussian distribution. It appears that the likelihood of velocity fluctuation increments in flight and in the wind tunnel are different. More precisely, it can be seen that the shape of the pdfs changes in a different way with an increase in turbulence intensity. In particular, for calm inflow conditions $\left(\varepsilon=5 \times 10^{-8} \mathrm{~m}^{2} / \mathrm{s}^{3}\right)$, the pdf corresponding to the wind tunnel inflow exhibits a Gaussian distribution, while the pdf corresponding to the flight inflow shows a slightly higher "peakedness" than a Gaussian distribution. Most importantly, for inflow conditions going from calm to lightly turbulent ( $\varepsilon$ increasing up to $1.1 \times 10^{-3} \mathrm{~m}^{2} / \mathrm{s}^{3}$ ), the "peakedness" of the probability density functions corresponding to the flight inflow increases and the pdfs differ strongly from the Gaussian distribution. However, considering the wind tunnel inflow, the "peakedness" increases only slightly for a dissipation rate $\varepsilon$ increasing from $\varepsilon=5 \times 10^{-8} \mathrm{~m}^{2} / \mathrm{s}^{3}$ to $\varepsilon=2 \times 10^{-5} \mathrm{~m}^{2} / \mathrm{s}^{3}$. The pdf then stays constant while the turbulence further increases (up to $\varepsilon=1.1 \times 10^{-3} \mathrm{~m}^{2} / \mathrm{s}^{3}$ ).

This discrepancy in the evolution of the probability density distributions of the velocity fluctuations means that there is a larger probability of higher velocity fluctuation increments in the wind tunnel inflow compared to the flight one. Moreover, for the flight inflow, the probability corresponding to large velocity increments decreases with an increase in turbulence level, but not for the wind tunnel inflow. This discrepancy in the probability density distributions of velocity fluctuations may be one reason for the different response of the transition location to increasing turbulence level observed between the flight measurements and the wind tunnel measurements. However, the exact physical mechanism is not yet clarified and it cannot be excluded that other aspects could impact the laminar-turbulent transition. In particular, it is well known that acoustic receptivity can drive transition (see for example [45]), and potential discrepancies between acoustic environments (for instance, possible tonal noise produced by glider components) have not been addressed.

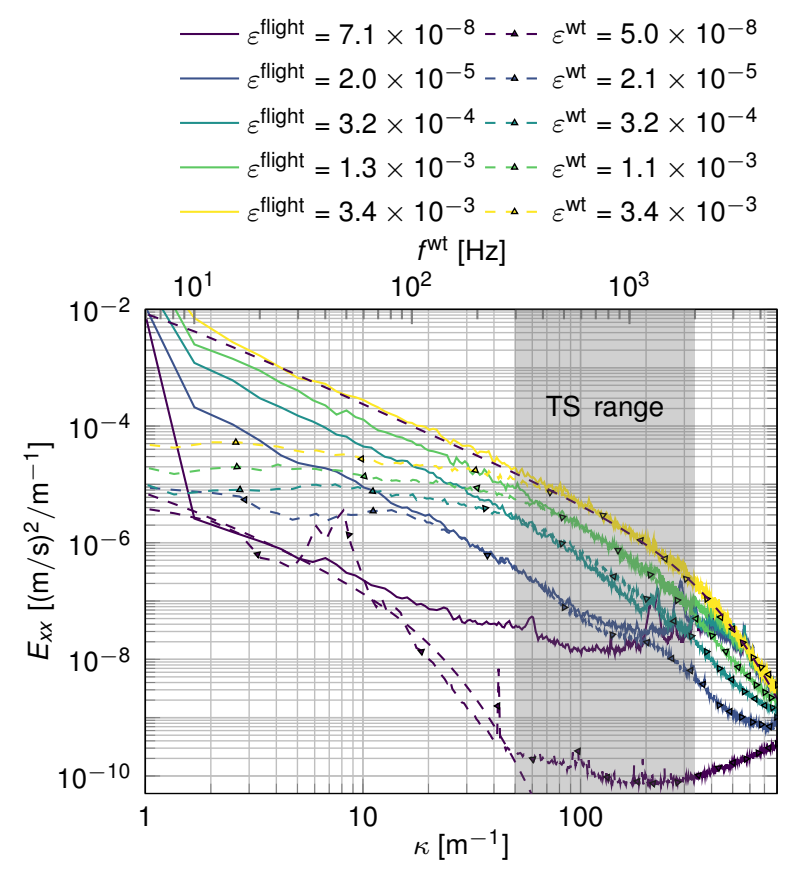

Fig. 7 Comparison between flight and wind tunnel one-dimensional turbulent kinetic energy spectra. 


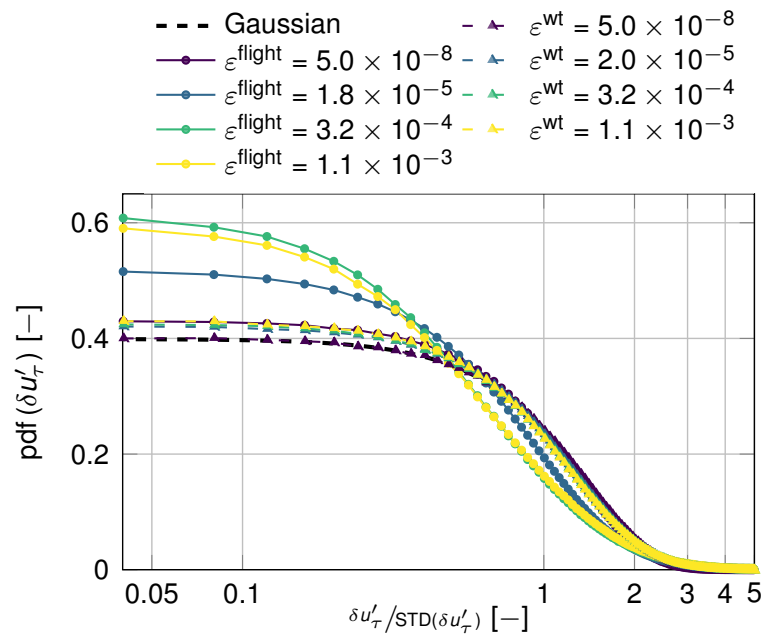

Fig. 8 Comparison between flight and wind tunnel pdfs. The selected time scales correspond to $1 / \tau=1000 \mathrm{~Hz}$ and $1 / \tau=750 \mathrm{~Hz}$ for the flight and the wind tunnel results, respectively.

\section{Conclusion}

The laminar-turbulent transition occurring along the pressure side of a laminar sailplane airfoil Flight conditions ranging from calm to moderately turbulent are reported using one and two point statistics, these being compared with results from Kolmogorov's' similarity theory. From these comparisons, it was shown how the energy dissipation rate, the energy spectrum and the probability density function of the velocity fluctuations can be estimated from the turbulence level. Then, for calm inflow conditions, it was demonstrated that the laminar-turbulent transition investigated in the context of this study was driven by TS-waves. Finally, the effects on the transition location of an increase in the inflow turbulence intensity from calm to lightly turbulent conditions were studied. Flight results showed a weak sensitivity of the transition location to an increase of turbulence level, unless it is associated with a modification of the incidence angle (modification of the pressure gradient). However, the sensitivity observed in the wind tunnel experiments, where the turbulence increase is generated using an active pneumatic grid, was significantly higher. It was argued that the differences in the response to turbulence could be due to the different probability density distributions of the velocity fluctuations of the inflow experienced in flight and in the wind tunnel.

\section{Funding Sources}

The authors are grateful to the Bundesministerium für Wirtschaft und Energie for financial support of this project through the Luftfahrtprogramm V project "Laminar-turbulente Transition unter instationären Anströmbedingungen" (LTTTUDA Förderkennzeichen 20E1503A, LTT-USTUTT Förderkennzeichen 20E1503B and LTT-USTUTT Förderkennzeichen 20E1503C). The work of Dr. Amandine Guissart was partly supported by the TU Darmstadt through its 'Future Talents' Short-term Scholarship and its Career Bridging Grant.

\section{Acknowledgments}

The authors thank Dennis Patzig from Akaflieg Darmstadt and the SLA Institute workshop for support with the construction of the experimental setup and are very appreciative of the assistance from the pilots, M. Stenger, T. Hofmann, W. Friedrichs and M. Jenkner.

\section{References}

[1] Weismüller, M., "A New Approach to Aerodynamic Performance of Aircraft under Turbulent Atmospheric Conditions," Ph.D. thesis, Technische Universität, Darmstadt, 2012.

[2] Reeh, A. D., and Tropea, C., "Behaviour of a natural laminar flow aerofoil in flight through atmospheric turbulence," Journal of Fluid Mechanics, Vol. 767, 2015, p. 394-429. https://doi.org/10.1017/jfm.2015.49. 
[3] Smith, A. M. O., and Gamberoni, N., "Transition, Pressure Gradient and Stability Theory.” Tech. Rep. ES-26388, Douglas Aircraft Company, 1956.

[4] van Ingen, J., "A suggested semi-empirical method for the calculation of the boundary layer transition region," Report, TU Delft (Technische Hogeschool Delft), 1956.

[5] Mack, L. M., “Transition and laminar instability,” Technical report, Jet Propulsion Lab., California Inst. of Tech.; Pasadena, CA, United States, 1977.

[6] Zanin, B. Y., "Transition at Natural Conditions and Comparison with the Results of Wind Tunnel Studies," Laminar-Turbulent Transition, edited by V. V. Kozlov, Springer Berlin Heidelberg, Berlin, Heidelberg, 1985, pp. 541-546. https://doi.org/10.1007/ 978-3-642-82462-3_67.

[7] Nitsche, W., Suttan, J., Becker, S., Erb, P., Kloker, M., and Stemmer, C., "Experimental and numerical investigations of controlled transition in low-speed free flight," Aerospace Science and Technology, Vol. 5, No. 4, 2001 , pp. 245 - 255. https://doi.org/10.1016/S1270-9638(01)01105-1.

[8] Erb, P., “Untersuchung der Grenzschichttransition im Flugversuch.” Ph.D. thesis, Technische Universität Darmstadt, 2002.

[9] Peltzer, I., "Flug- und Windkanalexperimente zur räumlichen Entwicklung von Tollmien-Schlichting-Instabilitäten in einer Flügelgrenzschicht," Ph.D. thesis, Technische Universität Berlin, Berlin, 2004.

[10] Seitz, A., and Horstmann, K., "In-Flight Investigations of Tollmien-Schlichting Waves,” IUTAM Symposium on One Hundred Years of Boundary Layer Research: Proceedings of the IUTAM Symposium held at DLR-Göttingen, Germany, August 12-14, $\underline{2004}$, edited by G. Meier, K. Sreenivasan, and H.-J. Heinemann, Springer, 2006, pp. 115-124. https://doi.org/10.1007/978-14020-4150-1_11.

[11] Duchmann, A., Simon, B., Tropea, C., and Grundmann, S., "Dielectric Barrier Discharge Plasma Actuators for In-Flight Transition Delay,” AIAA Journal, Vol. 52, No. 2, 2014, pp. 358-367. https://doi.org/10.2514/1.J052485.

[12] Saric, W. S., West, D. E., Tufts, M. W., and Reed, H. L., "Experiments on Discrete Roughness Element Technology for Swept-Wing Laminar Flow Control,” AIAA Journal, Vol. 57, No. 2, 2019, pp. 641-654. https://doi.org/10.2514/1.J056897.

[13] Kendall, J., "Boundary layer receptivity to freestream turbulence," 21st Fluid Dynamics, Plasma Dynamics and Lasers Conference, 1990. https://doi.org/10.2514/6.1990-1504.

[14] Horstmann, K., Quast, A., and Redeker, G., "Flight and wind-tunnel investigations on boundary-layer transition," Journal of Aircraft, Vol. 27, No. 2, 1990, pp. 146-150. https://doi.org/10.2514/3.45910.

[15] Reeh, A., Weismüller, M., and Tropea, C., "In-Flight Investigation of Transition Under Turbulent Conditions on a Laminar Wing Glove,” AIAA Journal, Vol. 52, No. 10, 2014, pp. 2176-2189. https://doi.org/10.2514/1.J052818.

[16] Bunker, A. F., "Turbulence and shearing stresses measured over the north atlantic ocean by an airplane-acceleration technique," Journal of Meteorology, Vol. 12, No. 5, 1955, pp. 445-455. https://doi.org/10.1175/1520-0469(1955)012<0445: TASSMO $>2.0 . \mathrm{CO} ; 2$.

[17] MacCready Jr., P. B., “Turbulence measurements by sailplane,” Journal of Geophysical Research (1896-1977), Vol. 67, No. 3, 1962, pp. 1041-1050. https://doi.org/10.1029/JZ067i003p01041.

[18] Payne, F. R., and Lumley, J. L., "One-dimensional spectra derived from an airborne hot-wire anemometer," Quarterly Journal of the Royal Meteorological Society, Vol. 92, No. 393, 1966, pp. 397-401. https://doi.org/10.1002/qj.49709239309.

[19] Lenschow, D. H., and Johnson, J., Warren B., "Concurrent Airplane and Balloon Measurements of Atmospheric Boundary-Layer Structure over a Forest," Journal of Applied Meteorology, Vol. 7, No. 1, 1968, pp. 79-89. https://doi.org/10.1175/15200450(1968)007<0079:CAABMO>2.0.CO;2.

[20] Sheih, C. M., Tennekes, H., and Lumley, J. L., "Airborne Hot-Wire Measurements of the Small-Scale Structure of Atmospheric Turbulence,” The Physics of Fluids, Vol. 14, No. 2, 1971, pp. 201-215. https://doi.org/10.1063/1.1693416.

[21] Rose, W. C., and Otten, I., L. J., “Airborne measurement of atmospheric turbulence,” Electromagnetic Wave Propagation from Aircraft, 1980, pp. 493-514.

[22] Otten, L. J., Pavel, A. L., Rose, W. C., and Finley, W. E., "Atmospheric Turbulence Measurements from a Subsonic Aircraft," AIAA Journal, Vol. 20, No. 5, 1982, pp. 610-611. https://doi.org/10.2514/3.51118. 
[23] Riedel, H., and Sitzmann, M., "In-flight investigations of atmospheric turbulence," Aerospace Science and Technology, Vol. 2, No. 5, 1998, pp. 301 - 319. https://doi.org/10.1016/S1270-9638(98)80007-2.

[24] Cho, J. Y. N., Newell, R. E., Anderson, B. E., Barrick, J. D. W., and Thornhill, K. L., "Characterizations of tropospheric turbulence and stability layers from aircraft observations," Journal of Geophysical Research: Atmospheres, Vol. 108, No. D20, 2003. https://doi.org/10.1029/2002JD002820.

[25] Li, S., Tse, K., Weerasuriya, A., and Chan, P., "Estimation of turbulence intensities under strong wind conditions via turbulent kinetic energy dissipation rates," Journal of Wind Engineering and Industrial Aerodynamics, Vol. 131, 2014, pp. 1 - 11. https://doi.org/10.1016/j.jweia.2014.04.008.

[26] Bodini, N., Lundquist, J. K., and Newsom, R. K., "Estimation of turbulence dissipation rate and its variability from sonic anemometer and wind Doppler lidar during the XPIA field campaign," Atmospheric Measurement Techniques, Vol. 11, No. 7, 2018, pp. 4291-4308. https://doi.org/10.5194/amt-11-4291-2018.

[27] Ohno, D., Romblad, J., and Rist, U., "Laminar to turbulent transition at unsteady inflow conditions: Direct numerical simulations with small scale free-stream turbulence," New Results in Numerical and Experimental Fluid Mechanics XII, edited by A. Dillmann, G. Heller, E. Krämer, C. Wagner, C. Tropea, and S. Jakirlić, Springer International Publishing, 2020, pp. 214-224. https://doi.org/10.1007/978-3-030-25253-3_21.

[28] Romblad, J., Ohno, D., Nemitz, T., Würz, W., and Krämer, E., "Laminar to Turbulent Transition due to unsteady Inflow Conditions: Wind Tunnel Experiments at increased Turbulence Levels," Deutscher Luft- und Raumfahrtkongress 2018, Friedrichshafen, 2018, pp. 1-9. https://doi.org/10.25967/480138.

[29] Romblad, J., Ohno, D., Guissart, A., Würz, W., and Krämer, E., "Response of NLF Airfoils to Small Scale Turbulence," Proceedings of the ninth IUTAM Symposium on Laminar-Turbulent Transition, London, UK, 2019, 2020, pp. 1-11. Submitted for publication.

[30] Romblad, J., Ohno, D., Würz, W., and Krämer, E., "Laminar to turbulent transition at unsteady inflow conditions: Wind tunnel measurements at oscillating inflow angle," New Results in Numerical and Experimental Fluid Mechanics XII, edited by A. Dillmann, G. Heller, E. Krämer, C. Wagner, C. Tropea, and S. Jakirlić, Springer International Publishing, 2020, pp. 254-264. https://doi.org/10.1007/978-3-030-25253-3_25.

[31] Reeh, A. D., "Natural Laminar Flow Airfoil Behavior in Cruise Flight through Atmospheric Turbulence," Ph.D. thesis, Technische Universität, Darmstadt, 2014.

[32] Simon, B. J., "Active Cancellation of Tollmien-Schlichting Waves under Varying Inflow Conditions for In-Flight Application." Ph.D. thesis, Technische Universität, Darmstadt, 2017.

[33] Guissart, A., Romblad, J., Ohno, D., Nemitz, T., and Tropea, C., "Laminar-to-turbulent tansition at unsteady inflow conditions: Flight experiments, wind tunnel experiments and numerical simulations," Deutscher Luft- und Raumfahrtkongress 2019 , Darmstadt, 2019, pp. 1-9. https://doi.org/10.25967/490043, in press.

[34] Leclère, Q., Pereira, A., Finez, A., and Souchotte, P., "Indirect calibration of a large microphone array for in-duct acoustic measurements," Journal of Sound and Vibration, Vol. 376, 2016, pp. 48-59. https://doi.org/10.1016/j.jsv.2016.04.033.

[35] Bradshaw, P. (ed.), An Introduction to Turbulence and its Measurement, Thermodynamics and Fluid Mechanics Series, Pergamon, 1971. https://doi.org/10.1016/C2013-0-02451-6.

[36] Plogmann, B., and Würz, W., "Aeroacoustic measurements on a NACA 0012 applying the Coherent Particle Velocity method," Experiments in Fluids, Vol. 54, No. 7, 2013, p. 1556. https://doi.org/10.1007/s00348-013-1556-9.

[37] Drela, M., "XFOIL: An Analysis and Design System for Low Reynolds Number Airfoils," Low Reynolds Number Aerodynamics, edited by T. J. Mueller, Springer Berlin Heidelberg, Berlin, Heidelberg, 1989, pp. 1-12. https://doi.org/10.1007/978-3-64284010-4_1.

[38] Pope, S. B., Turbulent flows, IOP Publishing, 2000.

[39] Welch, P. D., "The use of fast Fourier transform for the estimation of power spectra: A method based on time averaging over short, modified periodograms," IEEE Transactions on Audio and Electroacoustics, Vol. 15, No. 2, 1967, pp. 70-73. https://doi.org/10.1109/TAU.1967.1161901.

[40] Wyngaard, J. C., Turbulence in the Atmosphere, Cambridge University Press, 2010. https://doi.org/10.1017/ CBO9780511840524. 
[41] Kolmogorov, A., "The local Structure of turbulence in incompressible viscous fluid for very large Reynolds numbers (In Russian),” Dokl. Akad. Nauk SSSR, Vol. 30, 1941, pp. 299-303.

[42] Chen, W. Y., "Energy Dissipation Rates of Free Atmospheric Turbulence," Journal of the Atmospheric Sciences, Vol. 31, No. 8, 1974, pp. 2222-2225. https://doi.org/10.1175/1520-0469(1974)031<2222:EDROFA>2.0.CO;2.

[43] Kraichnan, R. H., “On Kolmogorov's inertial-range theories,” Journal of Fluid Mechanics, Vol. 62, No. 2, 1974 , p. $305-330$. https://doi.org/10.1017/S002211207400070X.

[44] Petzold, R., and Radespiel, R., "Transition on a Wing with Spanwise Varying Crossflow Evaluated with Linear Stability Theory," 43rd Fluid Dynamics Conference, 2013. https://doi.org/10.2514/6.2013-2466.

[45] Placidi, M., Gaster, M., and Atkin, C. J., "Acoustic excitation of Tollmien-Schlichting waves due to localised surface roughness," Journal of Fluid Mechanics, Vol. 895, 2020, p. R5. https://doi.org/10.1017/jfm.2020.349. 